\title{
Chemical cauterisation of bleeding spiders
}

\section{Introduction}

Spider naevi are frequently seen in patients of chronic liver disease (CLD). These are usually asymptomatic; but rarely bleed secondary to scratching and deranged coagulation profile. We report a 32 year old patient of alcoholic cirrhosis who had recurrent bleeding from spiders over the face after scratching which required pressure bandage and multiple blood transfusions. The bleeding spiders on the face were managed with chemical cautery using 50\% trichloroacetic acid (TCA). A drop of TCA was applied on the central arteriole with tip of woodstick. Each bleeding spider required a single application. The spider's central arteriole became flat after 10 to 14 days of application (Photograph 1). Patient is currently under followup for last 4 months after chemical cauterization of the spiders without recurrence of bleeding.

\section{Disscussion}

The spiders can bleed after scratching due to rich blood supply to the central arteriole. The bleeding can be profuse with the altered coagulation profile in decompensated cirrhotics. The bleeding spiders have been managed in the past using electrocautery, liquid nitrogen therapy, stereotactic radiosurgery and laser. ${ }^{1,2}$ The primary mechanism of using these methods is to obliterate the central arteriole of the spider. We used 50\% TCA which is being used in other dermatological conditions like mollascum contagiosum, warts, milia, xanthelesma, pyogenic granulomas. It leads to coagulation necrosis of the lesions. TCA is cheap, easily available and an effective option. To conclude, we used 50\% trichloroacetic acid to obliterate bleeding spider angioma to prevent further episodes of bleeding from spiders in decompensated cirrhotics.

\author{
M. NIJHAWAN ${ }^{1}$ \\ V.K. GUPTA ${ }^{2}$ \\ A. SINGH ${ }^{1}$ \\ S. CHANDER ${ }^{2}$ \\ S. NIJHAWAN ${ }^{2}$ \\ A. MATHUR ${ }^{2}$
}

Correspondence: Dr. M Nijhawan Department of Dermatology, MG Medical college ${ }^{1}$, 